\title{
Joint Symposium
}

\section{European: Joint Symposium with hosting NPA: Psychiatry in Transition: the European Experience}

\section{JS001}

\section{Psychiatry in transition: towards a reconceptualization of mental health and mental health disorders}

S. Galderisi

University of Campania Luigi Vanvitelli, Department of Psychiatry, Naples, Italy

Mental disorders are one of the top public health challenges affecting about $25 \%$ of the population of Europe every year and accounting for just under $40 \%$ of the disability burden. While the awareness of the key role of mental health in the attainment of better quality of life of European citizens has increased, policies and investments in mental health care not always parallel this development. A shift from long-term institutional mental health care to community-based services has occurred in many countries. However, progress in this direction is uneven across Europe and available data show a high degree of heterogeneity.

The economic crisis of the last decade also brought about additional challenges. In many countries services went through a severe shortage of human and financial resources, and couldn't guarantee adequate prevention, treatment and rehabilitation plans any longer. They also largely failed to incorporate new societal developments, such as cultural diversities, humanitarian emergencies and other factors linked to mass displacement, or new addictions, in the organization of mental health services.

A close collaboration among policy makers at the European and National level, scientific and professional associations and representatives of European patient and family organizations is highly needed to promote partnership action, disseminate best practices, promote mental health and prevent mental illnesses, and fully integrate mental health into EU policies. The European Psychiatric Association, the main association representing psychiatry in Europe, is committed to play a pivotal role in this process.

Disclosure of interest.- The authors have not supplied a conflict of interest statement.

\section{European: Joint Symposium with hosting NPA: Psychiatry in Transition: the European Experience}

\author{
JS002 \\ Identity of psychiatry in Europe \\ P. Falkai \\ University of Munich, Psychiatry and Psychotherapy, Munich, \\ Germany
}

The EPA has undergone substantial structural changes in the last few years and has currently 2 councils, the council of the individual members as well as the Council pf the representatives of the National Psychiatric Associations. One of the important questions for the future is how the EPA can develop in future and will these two councils work closely together in promoting the idea of European Psychiatry.

The negative image of psychiatry is not only due to unfavourable communication with the media, but is basically a problem of selfconceptualization. Much can be improved. However, psychiatry will remain a profession with an exceptional position among the medical disciplines, which should be seen as its specific strength. Disclosure of interest.- The authors have not supplied a conflict of interest statement.

\section{European: Joint Symposium with hosting NPA: Psychiatry in Transition: the European Experience}

\section{JS003 \\ Transition in psychiatry: myths and reality \\ J. Samochowiec \\ Pomeranian Medical University, Psychiatry, Szczecin, Poland}

In the last decades, there has been rapid political changes that influenced socio-economic status and changing roles in family life. Mental health care is moving from hospitalisation towards community psychiatry. However, big hospitals couldn't be shut down at once. It is a process that must evolve organically. 
Poland has started an important process of change in their mental health strategy and the management of patients affected by mental disorders aimed at transitioning towards a new approach and organization of Mental Health services, namely a community-based model centered on the specific needs (both clinical and not) of the patient and the family.

This is part of the transition, and from the organisational perspective, there is still a lot of work to do. We are also facing a need for new ways of teaching psychiatry. Because of the changes in psychiatry, the older generation must learn new techniques, and we need to learn how to communicate with patients in a different environment.

Early Diagnosis and Early Treatment are fundamental for reducing the impacts of the diseases and accelerate the recovery of the patients. Patients and Families have a key role in all the phases of the management of mental disorders: from preventing, to assisting and supporting the recovery of patients within work and social environments.

Disclosure of interest.- The authors have not supplied a conflict of interest statement.

\section{European: Joint Symposium with hosting NPA: Psychiatry in Transition: the European Experience}

\section{$\mathrm{JS} 004$}

\section{Reform of psychiatry in Poland: if} we're going to do this it's now or never

A. Szulc

Medical University of Warsaw, Department of Psychiatry, Pruszkow, Poland

Psychiatry in Poland for a long time was based primarily on hospitalization. As Polish Psychiatric Association we are now focused on developing community and outpatient care. In July 2018 a pilot program of mental health centres in Poland was launched, which aims to bring a significant change to the Polish psychiatry and increase the availability of psychiatric care.

The Polish Psychiatric Association also conducts its own activities that aim to break stereotypes about mental illnesses and combat the stigmatization of people suffering from depression or schizophrenia. At the beginning of this year, we have spread the Positive Manifest of Polish Psychiatric Association, which aims to change the attitudes of Poles towards psychiatry, psychiatrists and people with mental disorders.

Disclosure of interest.- The authors have not supplied a conflict of interest statement.

\section{Clinical/Therapeutic: Joint Symposium with EBC: Parkinson's Disease and Psychiatry}

\section{JS005 \\ Depression: risk factor or early symptom in Parkinson disease? \\ A. Leentjens \\ Maastricht University Medical Center, Psychiatry, Maastricht, The Netherlands}

Introduction.- there is convincing evidence that the risk of being diagnosed with Parkinson's disease is increased in the years following a diagnosis of depressive disorder. This has led to hypotheses of a possible pathophysiological relation between the two.
Objectives.- To explore the possible relation between depression and Parkinson's disease

Methods.- Narrative review of literature.

Results.- Studies in Parkinson's disease and other chronic conditions point towards a complex interrelation between depression and these diseases. First: depression may precede a diagnosis of Parkinson's disease, but it is not a specific risk factor, since depression can also precede other diagnoses, such as dementia, stroke, vascular disease, diabetes and various forms of cancer. In addition, distressing syndromes other than depression, such as anxiety and pain, can also increase the likelihood of a diagnosis of Parkinson's disease. Evidence also shows that a diagnosis of Parkinson's disease itself predisposes to later depression. Finally, even though pathophysiological alterations that constitute a risk for depression occur in all Parkinson patients, only a minority of patients will develop a depression. These observations make it difficult to formulate a pertinent biobehavioral model of depression in Parkinson's disease. Conclusions.- The relation between depression and Parkinson's disease is bidirectional and complex. Research has largely focused on Parkinson-specific pathophysiological risk factors for depression. However, non-specific risk factors for depression in the general population, as well as psychological and contextual factors also have to be taken into account in order to come to a more comprehensive etiological model for depression in patients with Parkinson's disease.

Disclosure of interest.- The authors have not supplied a conflict of interest statement.

\section{Educational: Joint Symposium with ECNP: What Will be the Impact of Digital Technology on the Practice of Clinicians in Psychiatry?}

\section{JS006 \\ Shaping the future: using digital technologies to support new treatment pathways in psychiatry \\ G. Dawson \\ P1 vital LTD, Psychiatry, Oxford, United Kingdom}

It takes 4-6 weeks after starting antidepressant therapy before a physician can detect whether the treatment is working. More than $50 \%$ of patients fail to respond to the first antidepressant prescribed. As a result a number of changes in medications maybe required before an effective treatment is found. However, previous work has shown that antidepressants induce changes in the processing of emotional information shortly after treatment is initiated and they may predict future improvements in mood. In a European wide study we sought to determine whether antidepressant-induced changes in emotional processing could be implemented to guide treatment changes much earlier than conventional methods. In a pilot study we found that a machine learning derived algorithm that combined changes in emotional processing with information derived from the Quick Inventory of Depression (QID-SR ${ }_{16}$ ) questionnaire provided the best predictor of response. This algorithm was incorporated in the P1vital ${ }^{\circledR}$ Predicting Response to Depression Treatment (PReDicT) Test, an online app patient's use to complete tests and questionnaires at home and transfers their data securely to their treating physician for evaluation. Here we report interim compliance and acceptability data from the PReDicT study ${ }^{1}$ to determine whether early response to antidepressants can be used to guide changes in treatment that leads to better outcomes for patients. Initially results from $>550$ patients showed that within two weeks a dose increase was prescribed in $16 \%$ of patients when treatment was guided by PReDicT compared to $\sim 6 \%$ receiving TaU. Similarly after 34 days 
of treatment a switch to an alternative medication was higher $(\sim 10 \%)$ in patients guided by PReDicT than those in the TaU arm $(\sim 3 \%)$. At the end of the 8 week study period $>90 \%$ of patients had completed their primary assessments suggesting that compliance with the app is high. These initial results suggest that the PReDicT test significantly influences physician prescribing behaviour Disclosure of interest.- The authors have not supplied a conflict of interest statement.

\section{Educational: Joint Symposium with EUFAS: Cannabis and the Brain: an Update for Psychiatrists}

\section{JS007}

\section{Measuring quantity matters: the need for a standard joint unit}

A. Gual, H. Lopez, M. Balcells

Neurosciences Institute. Hospital Clínic. IDIBAPS, Addictions Unit. Psychiatry Department, Barcelona, Spain

Cannabis use has grown steadily worldwide and specially in western societies, where a clear trend towards its legalization can be seen. Most of the epidemiological studies report on frequency of use, but data on quantities consumed are usually lacking. From a Public Health perspective there is a need to identify and measure the risks associated to cannabis, but in order to do it properly it is essential to have reliable information not only on frequency of use, but specially on quantities used. As it happened previously with alcohol, the need for quantification has lead to the development of the concept of a 'Standard Joint Unit (SJU)'.

In order to define a SJU we have conducted a study in the city of Barcelona with a sample of 400 cannabis users from four different settings: university, cannabis clubs, leisure areas and mental health centers. Participants were asked to donate one joint prepared by them and to fill some ad hoc questionnaires, including the CAST (Cannabis Abuse Screening Test). 492 questionnaires and 315 valid donations were obtained.

Donors were mostly men (77\%), with a mean age of 29 years, with at least high school education (72\%) and single (75\%). 84\% used cannabis four or more times per week, with an average of 4,2 (SD $3,7)$ joints per day of use. They tend not to share joints (55\%), use more often marihuana (74\%) than hashish (24\%) and always mix it with tobacco.

We analyzed the samples for their content of $\Delta 9$ tetrahidrocannabinol (9THC) and cannabidiol (CBD). Marihuana samples had a median of $6,56 \mathrm{mg}$ of 9THC and $0,02 \mathrm{mg}$ of CBD. Hashish samples had a median of 7,94 $\mathrm{mg}$ of 9THC and 3,24 $\mathrm{mg}$ of CBD. By consensus it was agreed that a standard joint contains $7 \mathrm{mg}$ of 9THC.

From a clinical and epidemiological perspective, quantity and frequency of use are equally important. The standard joint is a simple tool to estimate quickly the amount of 9THC consumed. Similar studies are needed in different countries and cultures to test if our results can be generalized.

Disclosure of interest.- The authors have not supplied a conflict of interest statement.

\section{Educational: Joint Symposium with EUFAS: Cannabis and the Brain: an Update for Psychiatrists}

\section{JS08}

\section{Is it possible to measure brain disfunction in cannabis use disorders? The visomotor rotation task as a biomarker of cerebellum alterations \\ L. Miquel \\ Addiction Unit Hospital Clínic of Barcelona. IDIBAPS, Psychiatry. ICN, Barcelona, Spain}

The Cerebellum is the brain structure for adaptive control. It is well known that it is involved in prediction and error-based learning functions which are necessary for motor control. However, in the last few years there is growing evidence that it is involved in addiction and other domains like attention, language or social cognition. We are far from understanding the correlation between changes in cerebellum activity and addiction behavior. However, the interest in this particular structure is growing recently.

The Cerebellum has a huge density of endocannabinoid (CB1) receptors, thus cerebellar changes due to acute and chronic cannabis use (CCU) have been observed in molecular and neuroimaging studies. Assessment tools to detect brain dysfunction due to substance use and misuse are scarce. Specifically, no assessment tools have been developed to identify cerebellar dysfunction in CCU. Here we present the results of a new and promising tool: the visuomotor rotation task. The visuomotor rotation task(VRT) measures motor adaptation which is a cerebellum dependent function. Motor adaptation is necessary to enhance precision and accuracy in movements and occurs when a difference between the predicted and actual outcome exists. This particular cerebellar function is impaired in cerebellar ataxic patients that have suffered injury in the cerebellum. Differences in the visuomotor rotation task performance have been observed in these patients compared with healthy controls. Less accuracy was observed and increasing error to the endpoint target was seen in healthy subjects while performing the task. We designed a protocol to analyse differences in VRT performance between CCU and healthy controls. We recruited 20 chronic cannabis users that fulfilled DSM-V criteria for cannabis use disorder and 20 age and sex matched healthy controls. Although patients who use cannabis do not show clinical signs of ataxia, they perform the task differently than healthy controls (Peak drift was significantly smaller for CCUs $\left(20.6+\mid-6.4^{\circ}\right.$ vs. $27.4+\mid-11.6^{\circ}$; $t(32)=-2.05, p=0.049$, Cohen's $d=-0.74,95 \% \mathrm{CI}=[-1.54,-0.05])$, suggesting that there is an alteration in the motor adaptation of these subjects.

This simple task should be a promising tool to measure cerebellum dysfunction due to long-term cannabis use and can help clinicians to understand why those patients have problems to learn and adapt to the changing environment.

Disclosure of interest.- The authors have not supplied a conflict of interest statement. 


\section{Educational: Joint Symposium with EUFAS: Cannabis and the Brain: an Update for Psychiatrists}

\section{JS09}

\section{Heavy long-term cannabis use: the neurocognitive effects \\ J. Cousijn}

University of Amsterdam, Psychology, Amsterdam, The Netherlands

The effect of cannabis on brain and behaviour appears mixed. This may stem from differential harms associated with cannabis exposure and cannabis dependence. Theoretical models suggest that the strength of cannabis cue-induced motivational processes (e.g., craving, approach action tendencies, attentional bias, withdrawal symptoms) support escalation and continuation of cannabis use. However, the loss of control over use may specifically be linked to compromised behavioral control over these motivational processes. The past decade we conducted multiple prospective functional magnetic resonance imaging and behavioral studies in long term heavy cannabis users ( $\sim 5$ days per week for at least 2 years) with varying levels of cannabis use-related problems. We thereby aimed at unravelling unique and common neurocognitive mechanisms underlying cannabis use versus dependence. Heavy cannabis users showed stronger cannabis cue-induced craving and attentional bias compared to non-using controls. Within the group of heavy cannabis users, craving was a consistent predictor of use, problem severity and treatment outcome. Behavioral control related cognitive processes (e.g., working-memory, inhibition, decision-making) did not differ between heavy cannabis users and non-using control in any of the studies. However, in one study individual differences in functioning of the frontoparietal behavioral control network in the brain was linked to increased problem severity over time. These finding support a more general role for cue-induced motivational processes in cannabis use. Behavioral control may uniquely be linked to cannabis dependence, however, future studies are needed to confirm these findings.

Disclosure of interest.- The authors have not supplied a conflict of interest statement.

\section{Educational: Joint Symposium with EUFAS: Cannabis and the Brain: an Update for Psychiatrists}

JS010

\section{The CANDIS Program, a validated intervention to treat cannabis use disorders}

E. Hoch

Ludwig Maximilian University, Department of Psychiatry, Munich, Germany

CANDIS treatment program - a validated intervention for cannabis use disorders.

Introduction.- Cannabis is the most frequently used illicit substance in Europe and beyond. The number of treated patients with cannabis use disorders increases since many years.

Objective.- To develop a cannabis-specific treatment program for adolescents and adults with cannabis use disorders.

Methods.- Within a national cannabis treatment research program a manualised cannabis-specific intervention was developed. Motivational Enhancement Therapy (MET), Cognitive-Behavioral Therapy (CBT) and Problem-Solving Training are combined (10 individual sessions). In a first RCT, the program was tested among 122 participants. In a second multi-site RCT, it was translated to 11 addiction-treatment centers (276 participants). Assessments were conducted at baseline, during each therapy session, at posttreatment and after three and six-months.

Results.- In the first RCT, the AT-group had higher abstinence rates than the control group (AT: 49\%; DTC: $12.5 \%$; $p<0.003$; ITT-analysis). Frequency of cannabis use, severity of dependence, number of disability days and general psychopathology also improved significantly. In the second RCT, post-treatment abstinence was reported by $53.3 \%$ of AT patients. The rate of negative drug urine screenings was $45.9 \%$. In the DTC-group, self-reported abstinence was $22 \%, 17.7 \%$ of drug urine screenings were negative. Group difference was significant ( $p<0.001$; ITT-analysis). Further, AT patients improved in frequency of cannabis use, number of cannabis dependence criteria, severity of dependence, and number and severity of cannabis-related problems. Treatment retention was $88 \%$ and $65 \%$. Effects were stable in the follow-up assessments. Conclusions.- Both RCTs show, that participants benefit largely from the intervention.

Disclosure of interest.- The authors have not supplied a conflict of interest statement. 